\title{
Validation of the Oregon Scientific BPU 330 for self-monitoring of blood pressure according to the International Protocol
}

\author{
$\mathrm{Li} \mathrm{Li'}$ \\ XinYu Zhang' \\ ChunHong Yan' \\ QingXiang Liang ${ }^{2}$ \\ 'Biomedical Engineering Lab, Faculty \\ of Information Engineering, ShenZhen \\ University, ShenZhen, China; ${ }^{2}$ Bao An \\ People's Hospital, ShenZhen, China
}

Correspondence: XinYu Zhang Biomedical Engineering Lab, Faculty of Information Engineering, ShenZhen University, ShenZhen, China

Tel +8675526534207

Fax +86 75526534314

Email lucyyan.wu@gmail.com

\begin{abstract}
Objective: Extensive marketing of devices for self-measurement of blood pressure has created a need for purchasers to be able to satisfy themselves that such devices have been evaluated according to agreed criteria. The Oregon Scientific BPU 330 blood pressure monitor is an electronic device for upper arm measurement. This study assessed the accuracy of the Oregon Scientific BPU 330 blood pressure monitor according to the International Protocol by the Working Group on Blood Pressure Monitoring of the European Society of Hypertension for validation of blood pressure measuring devices.

Method: 52 participants over 30 years of age were studied in the validation. Nine blood pressure measurements were taken alternately with a mercury sphygmomanometer by two observers, and by the supervisor, using the BPU 330 device. A total of 33 participants were selected for the analysis. The validation was divided into two phases. Phase 1 included 15 participants. If the device passed phase 1, 18 more participants were included. The 99 pairs of measurements were compared according to the International Protocol. The device was given a pass/fail recommendation based on its accuracy compared with the mercury standard (within 5, 10, and $15 \mathrm{mmHg}$ ), as well as the number met in the ranges specified by the International Protocol.

Results: The mean and standard deviation of the difference between the mean of the observers and the BPU 330 device were $1.7 \pm 4.7 \mathrm{mmHg}$ and $2.8 \pm 3.9 \mathrm{mmHg}$ for systolic blood pressure (SBP) and diastolic blood pressure (DBP), respectively. In phase 1, the device passed with a total of 33, 43, and 44 SBP readings; 38, 44, and 45 DBP readings were within 5, 10, and $15 \mathrm{mmHg}$, respectively. In phase 2.1, 81, 95, and 96 for SBP, and 83, 95, and 98 for DBP readings fell within the zones of 5, 10, and $15 \mathrm{mmHg}$, respectively. In phase 2.2, the last phase, 28 participants fell within the zone of two of the three comparisons, lying within $5 \mathrm{mmHg}$ for SBP and 29 participants for DBP. No participants fell within the zone of all three of their comparisons over $5 \mathrm{mmHg}$ apart for both SBP and DBP.
\end{abstract}

Conclusion: The BPU 330 can be recommended for self-monitoring of blood pressure in the adult population, according to the International Protocol.

Keywords: blood pressure, self-monitoring, hypertension, International Protocol

\section{Introduction}

Hypertension is an important public health challenge worldwide because of its high prevalence and the concomitant increase in risk of disease. It is the most important risk factor for cardiovascular, cerebrovascular, and renal disease (Kearney et al 2004). Self-monitoring of blood pressure (BP) is regarded as a useful adjunct to conventional office BP measurements (Stergiou et al 2007). Indeed, self-monitoring can provide valuable information on how the BP of patients under treatment is being controlled, and can improve patient compliance with antihypertension therapy (Assaad et al 2003). Extensive marketing of automated and semi-automated devices to manage BP enables 
accurate and repeated BP measurements, which in turn aids in the identification of patients suffering from high BP (Pini et al 2007). In 2002, the Working Group on Ambulatory Blood Pressure Monitoring of the European Society of Hypertension released its International Protocol for validation of BP-measuring devices in adults (O'Brien et al 2002). In comparison with the Association for the Advancement of Medical Instrumentation (AAMI 1993) and the British Hypertension Society (BHS; O'Brien et al 1993), the International Protocol has been simplified in terms of sample size required and entry $\mathrm{BP}$ range. We used the International Protocol to perform a validation study of the Oregon Scientific BPU 330 (Oregon Scientific, Tualatin, USA), an upper arm BP monitor.

\section{Method}

\section{Test device}

The Oregon Scientific BPU330 is an upper arm BP monitor, which uses the oscillometric method of BP measurement. It measures BP at rest ranging between 30 and $280 \mathrm{mmHg}$, and pulse rate between 40 and 200 beats/min. Inflation is performed by an automatic electric pumping system, and deflation by automatic pressure-release valve. The device has a large liquid crystal display to show systolic and diastolic $\mathrm{BP}$ and heart rate and a memory capacity of 14 readings. It weighs about $340 \mathrm{~g}$ without batteries and measures $128 \times 115$ $\times 64 \mathrm{~mm}$. An arm cuff suitable for arm circumferences of 22 to $42 \mathrm{~cm}$ is supplied with the device. The device is powered by four $1.5 \mathrm{~V}$ batteries or an $\mathrm{AC}$ adaptor. A particular feature is the talking function to give voice instructions on how to use the device. After each measurement, the device reads out the BP value and interprets BP classification according to World Health Organization (WHO) and International Society of Hypertension (ISH) guidelines (ISH 1999).

\section{Participants}

A total of 52 participants were measured in the validation study. According to the International Protocol, 33 participants were selected within the ranges of BP required in each category. Participants with arterial fibrillation and arrhythmias were excluded from the validation, whereas participants currently on pharmacological treatment for hypertension were included. Demographic data including age, sex, arm circumference, weight, height, and preexisting diseases were recorded. Table 1 shows the participant number distributed in each BP range.

\section{Procedure}

One supervisor and two observers trained as described in Appendix B of the International Protocol conducted the validation study. The observers simultaneously measured BP using the auscultatory technique with standard mercury sphygmomanometer; the supervisor checked the values obtained by the observers and measured the BP with the test device.

The participant was introduced to the observers and the procedure explained. BP was measured in a warm quiet room. Arm and wrist circumference, sex, date of birth, and current date and time were noted. The participant was then asked to relax for 10-15 mins. BP was taken by the observers with a standard sphygmomanometer using a homologated two-headed binaural stethoscope. The supervisor measured BP with the test device, and checked the agreement of the measurement taken by the observers. The supervisor and observers were blinded to each other's reading. Observers' readings with a difference greater than $4 \mathrm{mmHg}$ were repeated until closer agreement was reached. The aforesaid same measurements were not taken more than three times. All measurements were taken on the left arm, which was supported at heart level. At least 30 seconds were allowed between each measurement to avoid venous congestion, but no more than 60 seconds, to avoid increasing variability.

Nine sequential same-arm measurements using the test instrument and a standard mercury sphygmomanometer were recorded as follows:

BPA: Entry BP was taken by observers 1 and 2 each with the mercury standard. The mean values were used to categorize the participant into a low, medium, or high range separately for SBP and DBP (Table 1).

BPB: Device detection BP taken by the supervisor. This measurement was taken to allow the test instrument to determine the BP characteristics of the participant. More than one attempt may be needed with some devices; this measurement was not included in the analysis. If the device failed to record a measurement after three attempts, the participant was excused.

BP1: Observers 1 and 2 with the mercury standard.

BP2: Supervisor with the test instrument.

BP3: Observers 1 and 2 with the mercury standard.

BP4: Supervisor with the test instrument.

BP5: Observers 1 and 2 with the mercury standard.

BP6: Supervisor with the test instrument.

BP7: Observers 1 and 2 with the mercury standard.

\section{Analysis}

For assessment of accuracy, only measurements BP1 to BP7 were used. The mean of each pair of observer measurements 
Table I Classifi cation of participants according to BP level

\begin{tabular}{lllll}
\hline & SBP $(\mathbf{m m H g})$ & DBP $(\mathbf{m m H g})$ & \multicolumn{2}{l}{ Participants $(\mathbf{n})$} \\
\cline { 3 - 5 } & & & Phase I & Phase 2 \\
\hline Low & $90-129$ & $40-79$ & 5 & $1 \mathrm{I}$ \\
Medium & $130-160$ & $80-100$ & 5 & $\mathrm{II}$ \\
High & $161-180$ & $101-130$ & 5 & $1 \mathrm{I}$ \\
\hline
\end{tabular}

Abbreviations: SBP, systolic blood pressure; DBP, diastolic blood pressure.

was calculated; these were denoted as observer measurements BP1, BP3, BP5, and BP7. Each device measurement was flanked by two of these observer measurements. One of these was selected as the comparative measurement.

From these, further measurements were derived as follows.

1. The differences between BP2 and BP1 (BP2-BP1), BP2BP3, BP4-BP3, BP4-BP5, BP6-BP5, and BP6-BP7 were calculated.

2. The absolute values of the differences were calculated.

3. These were paired according to the device reading.

4. If the values in a pair were unequal, the observer measurement corresponding to the smaller difference was used.

5. If the values in a pair were equal, the first of the two observer measurements was used.

When this analysis was completed, there were three device readings for SBP and three for DBP for each participant.

The differences were classified into three zones (within 5, 10, and $15 \mathrm{mmHg}$ ), separately for SBP and DBP, for 15 participants in phase 1 and all 33 participants in phase 2.1. For phase 2.2, the number of differences of less than
Table 2 Characteristics of the study participants $(n=33)$

\begin{tabular}{lll}
\hline Characteristics & Mean \pm SD & Range \\
\hline Age (years) & $46 \pm 13$ & $30-78$ \\
Arm circumferences $(\mathrm{cm})$ & $36 \pm 4$ & $25-43$ \\
Weight $(\mathrm{kg})$ & $68 \pm 14$ & $48-96$ \\
Height $(\mathrm{cm})$ & $169 \pm \mathrm{II}$ & $155-186$ \\
Pulse rate (beat per $\mathrm{min})$ & $77 \pm 1 \mathrm{I}$ & $61-108$ \\
Mean systolic pressure $(\mathrm{mmHg})$ & $144 \pm 31$ & $90-179$ \\
Mean diastolic pressure $(\mathrm{mmHg})$ & $88 \pm 18$ & $54-122$ \\
\hline
\end{tabular}

$5 \mathrm{mmHg}$ determined a pass/fail grade for 33 participants, and determined a pass/fail recommendation (Table 3 ). If the device failed at the first phase, the validation was complete. To pass the validation and gain recommendation, a device had to pass both phase 2.1 and 2.2.

\section{Result}

A total of 52 participants were measured in the study. Participants excluded consisted of 3 whose Korotkoff sound was too weak, 5 who opted out of the study before a complete measurement, 2 for whom the test BPU 330 gave repeat error readings, and 6 whose BP measurements were outside the $\mathrm{BP}$ ranges defined in the inclusion criteria. Three participants were excluded because the protocol-specified BP category was already full. The characteristics of the 33 participants enrolled are shown in Table 2. The average age of the participants was $46 \pm 13$ years (19 men and 14 women). The upper arm circumference was $36 \pm 4 \mathrm{~cm}$. The auscultatory BP measurements lay in the range $90-179 \mathrm{mmHg}$ for SBP and 54-122 $\mathrm{mmHg}$ for DBP.

Table 3 Result of BPU 330

\begin{tabular}{|c|c|c|c|c|c|c|c|}
\hline Phase I & & $\leq 5 \mathrm{mmHg}$ & $\leq 10 \mathrm{mmHg}$ & $\leq 15 \mathrm{mmHg}$ & Recomm. & & \\
\hline Required & One of & 25 & 35 & 40 & & & \\
\hline \multirow[t]{2}{*}{ Achieved } & SBP & 33 & 43 & 44 & Continue & & \\
\hline & DBP & 38 & 44 & 45 & Continue & & \\
\hline Phase 2.1 & & $\leq 5 \mathrm{mmHg}$ & $\leq 10 \mathrm{mmHg}$ & $\leq 15 \mathrm{mmHg}$ & Recomm. & MD & SD \\
\hline \multirow[t]{2}{*}{ Required } & Two of & 65 & 80 & 95 & & & \\
\hline & All of & 60 & 75 & 90 & & & \\
\hline \multirow[t]{2}{*}{ Achieved } & SBP & 81 & 95 & 96 & Pass & 1.7 & 4.7 \\
\hline & DBP & 82 & 95 & 98 & Pass & 2.8 & 3.9 \\
\hline Phase 2.2 & & $2 / 3 \leq 5 \mathrm{mmHg}$ & $0 / 3 \leq 5 \mathrm{mmHg}$ & & Recomm. & & \\
\hline Required & & $\geq 22$ & $\leq 3$ & & & & \\
\hline \multirow[t]{2}{*}{ Achieved } & SBP & 28 & 0 & & Pass & & \\
\hline & DBP & 29 & 0 & & Pass & & \\
\hline
\end{tabular}

Abbreviations: Recomm, recommendation; $\mathrm{MD}$, mean difference $(\mathrm{mmHg})$; $\mathrm{SD}$, standard deviation $(\mathrm{mmHg})$. 
As shown in Table 3 , a total of 33,43 , and 44 SBP readings and 38, 44, and 45 DBP readings in phase 1 were within 5, 10, and $15 \mathrm{mmHg}$, respectively. The device passed the requirements of phase 1 in the study group. In phase 2.1, 99 sets of measurements were available for analysis. In a sample of 33 participants, 81, 95, and 96 for SBP and 82, 95, and 98 for DBP were within 5, 10, and $15 \mathrm{mmHg}$, respectively.

In the analysis conducted for the last phase (phase 2.2), 28 participants had at least two of the differences within $5 \mathrm{mmHg}$ for SBP and 29 participants had at least two of the differences within $5 \mathrm{mmHg}$ for DBP. None fell in the zone of all three of their comparisons over $5 \mathrm{mmHg}$ apart for both SBP and DBP.
Thus the last phase was also successfully completed. The means and standard deviations of the difference between the means of the observers and BP device were $1.7 \pm 4.7 \mathrm{mmHg}$ and $2.8 \pm 3.9 \mathrm{mmHg}$ for SBP and DBP, respectively.

Bland-Altman plots of device-observer differences against the mean BP of device and observers are shown in Figure 1 for SBP and DBP, respectively.

\section{Discussion}

In recent years, self-monitoring of BP by patients at home is being increasingly used in clinical practice (Stergiou et al 2007). Patients and doctors increasingly use automatic
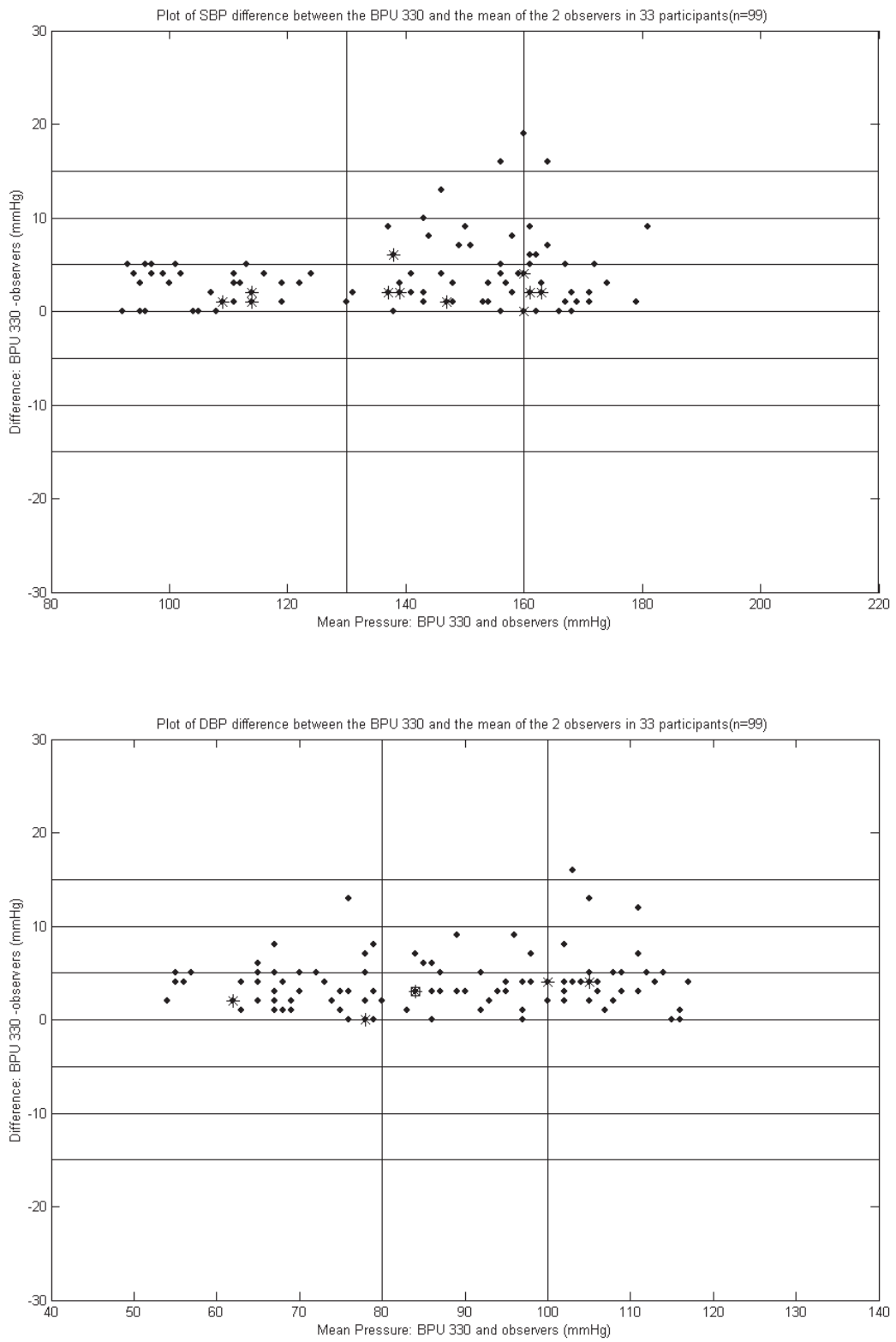

Figure I Bland-Altman plots for systolic blood pressure (SBP) and diastolic blood pressure (DBP) (means of observers and device readings) versus the difference between the BPU 330 and the mercury sphygmomanometer.

Note: The stars indicate two measurements ; the square dots indicate three measurements. 
devices for home BP monitoring (HBPM) (Omboni et al 2007). HBPM has been shown to improve patient BP control, inform treatment decisions, and provide diagnostic as well as prognostic information (Viera and Hinderliter 2007). Moreover, HBPM is free of the white-coat effect, can detect the masked hypertension phenomenon and, compared to office BP measurements, is more reproducible, is correlated more closely to target organ damage, and better predicts cardiovascular events (Braam et al 2004; Greeff and Shennan 2007). For this reason, the wide use of BP monitors at home is a consequence of the high value of self-monitoring of BP. Validation, accuracy assessment, and precision testing are still essential for each new device entering the market.

This study provides information on the accuracy and reliability of the Oregon Scientific BPU 330 for self-monitoring of BP. It showed that the device comfortably fulfilled the validation requirements in accordance with the International Protocol. The BPU 330 device was accurate for both SBP and DBP, passing all phases of the International Protocol.

The average device-observer difference was lower than $3 \mathrm{mmHg}$ (1.7 for SBP and 2.8 for DBP) and the standard deviation of the device-observer difference was never greater than $5 \mathrm{mmHg}$ (4.7 for SBP and 3.9 for DBP), indicating that the device is highly accurate.

The analysis shows that the BPU 330 provides accurate and reliable BP measurements and is capable of generating precise readings across a wide spectrum of participants. BPU 330 can give voice instructions and provide memory. Each of these can help the user to record the BP history readings with date and time stamps, with the voice function. We therefore recommend that BPU 330 can be used as a home BP monitoring device for hypertension.

The BPU 330 can be recommended for self-monitoring of BP in the adult population according to the International Protocol.

\section{Acknowledgments}

The device used in this study was donated by IDT Technology Ltd (Hong Kong, China). The authors report no conflicts of interest in this work.

\section{References}

[AAMI] Association for the Advancement of Medical Instrumentation. 1993. American National Standard: Electronic or Automated sphygmomanometer. Arlington, VA: AAMI.

Assaad MAE, Topouchian JA, Asmar RG. 2003. Evaluation of two devices for self-measurement of blood pressure according to the international protocol: the Omron M5-I and the Omron 705IT. Blood Press Monit, $8: 127-33$.

Braam RL, Aslan B, Thien T. 2004. Accuracy of the Omron RX-M, and automated blood pressure measuring device, measuring blood pressure at the wrist, according a modified British Hypertension Society protocol. Blood Press Monit, 9:25-30.

Greeff AD, Shennan AH. 2007. The health and Life device (HL888HA): accuracy assessment in an adult population according to the British hypertension society protocol. Blood Press Monit, 12:107-11.

[ISH] International Society of Hypertension. 1999. International Society of Hypertension Guidelines for the management of hypertension. J Hypertension, 17:151-83.

Kearney PM, Whelton M, Rernolds K, et al. 2004. Worldwide prevalence of hypertension: a systematic review. J Hypertension, 22:11-19.

O'Brien E, Petrie J, Littler WA, et al. 1993. The British Hypertension Society protocol for the evaluation of blood pressure measuring devices. J Hypertension, 11(Suppl 2):S43-S62.

O'Brien E, Pickering T, Asmar R, et al. 2002. Working Group on Blood Pressure Monitor of the European Society of Hypertension International Protocol for validation of blood pressure measuring devices in adults. Blood Press Monit, 7:3-17.

Omboni S, Riva I, Giglibo A, et al. 2007. Validation of Omron M5-I, R5-I and HEM-907 automated blood pressure monitors in elderly individuals according to the International Protocol of the European Society of Hypertension. Blood Press Monit, 12:133-42.

Pini C, Pastori M, Baccheschi J, et al. 2007. Validation of the Artsana CSI 610 automated blood pressure monitor in adults according to the International Protocol of the European Society of Hypertension. Blood Press Monit, 12:179-84.

Stergiou GS, Bernd J, Giovas PP, et al. 2007. Validation of the Microlife WatchBP home device for self home blood pressure measurement according to International Protocol. Blood Press Monit, 12:185-8.

Stergiou GS, Jaenecke B, Giovas PP, et al. 2007. A tool for reliable self-home blood pressure monitoring designed according to European Society of Hypertension recommendation: the Microlife WatchBP home monitor. Blood Press Monit, 12:127-31.

Viera AJ, Hinderliter AL. 2007. Validation of the HEM-780REL with easy wrap cuff for self-measurement of blood pressure according to the European Society of Hypertension International Protocol. Blood Press Monit, 12:335-8. 
